Mathématiques et sciences humaines
Mathematics and social sciences

179 | Automne 2007

Varia

\title{
La constitution du concept de composé partitionnel chez D. Foata : éléments d'une épistémologie de la combinatoire
}

The constitution of the concept of "composé partitionnel" in D. Foata : elements

of an epistemology of combinatorics

\section{Michel Serfati}

\section{OpenEdition}

Journals

Édition électronique

URL : http://journals.openedition.org/msh/7193

DOI : $10.4000 /$ msh.7193

ISSN : $1950-6821$

Éditeur

Centre d'analyse et de mathématique sociales de l'EHESS

Édition imprimée

Date de publication : 1 septembre 2007

Pagination : 101-107

ISSN : 0987-6936

Référence électronique

Michel Serfati, « La constitution du concept de composé partitionnel chez D. Foata : éléments d'une épistémologie de la combinatoire ", Mathématiques et sciences humaines [En ligne], 179 | Automne 2007, mis en ligne le 21 décembre 2007, consulté le 23 juillet 2020. URL : http:// journals.openedition.org/msh/7193; DOI : https://doi.org/10.4000/msh.7193 


\title{
LA CONSTITUTION DU CONCEPT DE COMPOSÉ PARTITIONNEL CHEZ D. FOATA : \\ ÉLÉMENTS D'UNE ÉPISTÉMOLOGIE DE LA COMBINATOIRE
}

\author{
Michel SERFATI ${ }^{1}$
}

\begin{abstract}
RÉSUMÉ - Cet article d'épistémologie est consacré à décrire la constitution par Dominique Foata d'un concept (le composé partitionnel $\mathrm{Y}^{+}$d'une suite $\mathrm{Y}=\mathrm{Y}_{\mathrm{n}}$ d'ensembles finis) et d'une certaine figure de pensée mathématique corrélative. Tous deux sont progressivement construits à partir d'identités d'analyse bien connues, associées par exemple à la fonction génératrice des polynômes d'Hermite.

MOTS-CLÉS - Composé partitionnel, Foata, Fonction génératrice, Graphe involutionnaire, Mehler, Polynôme d'Hermite

SUMMARY - The constitution of the concept of composé partitionnel in D. Foata: elements of an epistemology of combinatorics

This epistemological paper is devoted a concept defined by Dominique Foata (the composé partitionnel $\mathrm{Y}^{+}$of a sequence $\mathrm{Y}=\mathrm{Y}_{\mathrm{n}}$ of finite sets) and to some associated mathematical themes of thought. Both are progressively introduced, starting from well known analytical identities, associated for instance to the generating function of Hermite polynomials.
\end{abstract}

KEY-WORDS - Composé partitionnel, Foata, Generating function, Hermite polynomials, Involutionary graph, Mehler

Le texte de Dominique Foata ci-dessus, Une méthode combinatoire pour l'étude des fonctions spéciales décrit la très remarquable élaboration d'une figure de pensée mathématique à partir de simples identités d'analyse.

Statistiques et graphiques à l'appui, l'auteur explique d'abord bien le développement considérable, qualitatif et quantitatif, des travaux et des publications en matière de combinatoire depuis les années 1960 - un célèbre article de Giancarlo Rota cité dans l'article ${ }^{2}$ servant ici de toile de fond à un bilan daté de 1993. Il est donc bien évidemment exclu qu'un article puisse couvrir à lui seul le champ si divers des méthodes en combinatoire : " Dresser le catalogue des méthodes est une tâche impossible », écrit donc justement l'auteur. Il s'agira donc de mettre en lumière une méthode seulement - composés partitionnels et formule exponentielle - cependant véritablement exemplaire comme on verra, et dont $\mathrm{D}$. Foata fut le créateur dans les années 1970-1980. Pour rédiger le présent commentaire épistémologique, nous nous

\footnotetext{
${ }^{1}$ Institut de Recherche sur 1'Enseignement des Mathématiques (IREM), Université Paris VII-Denis Diderot, serfati@math.jussieu.fr

${ }^{2}$ Cf. [Rota, 1996] dans l'article de D. Foata.
} 
sommes ainsi appuyés sur d'autres textes de l'auteur cités en bibliographie de son article, et tout particulièrement sur un remarquable opuscule La série génératrice exponentielle dans les problèmes d'énumération".

Si l'origine de cette recherche doit être incontestablement située dans diverses résultats d'analyse classiques depuis le $\mathrm{XIX}^{\mathrm{e}}$ siècle - les polynômes orthogonaux, d'Hermite en l'occurrence - D. Foata explique comment l'interprétation combinatoire nouvelle que lui-même a apportée à deux formules «hermitiennes» connues depuis longtemps, allait servir de paradigme épistémologique à une construction algébricocombinatoire générale, celle du composé partitionnel $Y^{+}$d'une suite $Y$ d'ensembles finis, en une démarche qui allait se dégager de la spécificité et de la contingence de l'exemple « hermitien » pour l'englober et le dépasser tout à la fois. Élaborée en effet en termes de graphes involutionnaires finis à partir de l'exemple support, la méthode des composés partitionnels allait en vérité trouver un champ d'application et susciter des questionnements de recherche dans de nombreuses autres structures combinatoires (ainsi des graphes finis quelconques, des applications entre ensembles finis, des permutations) pour devenir in fine une méta-méthode, qu'on peut ainsi légitimement décrire comme une figure de pensée combinatoire. Ainsi Foata écrit-il sur ce point

\begin{abstract}
Je vais ainsi parler d'une technique combinatoire, popularisée dans les années 70-80, qui a révolutionné la manière d'établir de nombreuses idéntités sur les fonctions spéciales et montrer comment cette approche s'écarte fondamentalement des méthodes classiques utilisées en analyse.
\end{abstract}

On distinguera trois temps logiques dans l'article, qui correspondent à autant de formules : celle d'abord de la génération des polynômes d'Hermite par une formule exponentielle. Ensuite, la formule « bilinéaire » de Mehler, toujours pour les polynômes d'Hermite. Enfin, la troisième, extension de la précédente, dite multilinéaire. La philosophie profonde du texte - le coeur de la méthode - est, nous semble-t-il, que, pour apparemment ancrés qu'ils soient dans l'analyse, ces résultats ne sont que des réalisations concrètes contingentes (des «expressions closes », nous dit le texte) d'une formule paradigmatique combinatoire qui les transcende et les «explique » tout à la fois, l'identité exponentielle.

Le texte fait intervenir continûment les polynômes d'Hermite $H_{n}(x)$. Rappelons que ceux-ci sont classiquement définis comme orthogonaux sur $]-\infty,+\infty[$ pour le poids

$$
\phi(x)=\exp \left(-\frac{x^{2}}{2}\right)
$$

Il y a bien d'autres définitions (classiques) de ces objets bien connus, qui sont citées et utilisées dans l'article. Ainsi peuvent-il être aussi créés à partir de leur fonction génératrice exponentielle

$$
\sum_{n \geq 0} \frac{u^{n}}{n !} H_{n}(x)=\exp \left(u x-\frac{u^{2}}{2}\right)
$$

Classiquement, l'injection dans l'argument de l'exponentielle de droite d'un paramètre réel «muet» de signe $x$ permet de récupérer les $H_{n}(x)$ comme autant de coefficients du développement en série - c'est la mécanique usuelle des fonctions

${ }^{3}$ Cf. [Foata, 1974] dans l'article de D. Foata. Aussi A combinatorial proof of the Mehler formula, [Foata, 1978]. 
génératrices, ici exponentielle. C'est cette formule (classique) qui va servir de point de départ à l'exposé - nous la dirons ici pivot. D'un autre côté, utilisant l'une ou l'autre des définitions, on peut aussi expliciter les $H_{n}(x)$ suivant

$$
H_{n}(x)=\sum_{0 \leq 2 k \leq n}(-1)^{k} x^{n-2 k} \frac{n !}{(n-2 k) ! 2^{k} k !}
$$

La seconde formule-clé du texte est alors la très ancienne (1866) formule « bilinéaire » de Mehler vérifiée par les $H_{n}(x)$ :

$$
\sum_{n \geq 0} \frac{u^{n}}{n !} H_{n}(x) H_{n}(y)=\left(1-u^{2}\right)^{-1 / 2} \exp \left(\frac{2 u x y-u^{2}\left(x^{2}+y^{2}\right)}{2\left(1-y^{2}\right.}\right)
$$

D. Foata présente alors naturellement la méthode à partir de l'exemple de base du pivot (1). Bien évidemment, il ne s'agit aucunement de redémontrer l'une ou l'autre de ces formules par un quelconque procédé analytique! Si dans un paragraphe historique du plus haut intérêt, l'auteur évoque néanmoins à leur sujet une liste de diverses démonstrations analytiques, il est bien clair que la visée combinatoire ne peut se situer dans ce registre. En vérité, le schéma méthodologique proprement combinatoire, consiste à réinterpréter chacun des deux termes de la formule pivot (1) comme provenant d'une énumération, et leur égalité comme la mise en regard (une adéquation) de deux façons distinctes de dénombrer un même ensemble support - une problématique d'essence profondément leibnizienne. On reconnaît ici une méthodologie combinatoire quotidienne. Pour énumérer, il faut donc préalablement mettre en place un cadre ensembliste, lequel n'est évidemment nullement fourni par le résultat brut (1) et c'est l'art du combinatoricien que de promouvoir et d'exhiber un tel support sous-jacent à la formule qui soit aussi adéquat à chacun de ses deux membres.

Comme l'explique bien l'auteur, le cadre ensembliste général sera, dans cet exemple spécifique, celui de la famille de toutes les involutions de tous les ensembles finis $[n]=\{1,2, \ldots, n\} \quad\left(n \in \mathbb{N}^{*}\right)$ - la donnée de cette famille étant à l'évidence équivalente à celle de tous leurs graphes, dits involutionnaires. Le résultat, immédiat d'après sa factorisation en cycles, mais central dans la suite, est qu'une telle involution se constitue à partir de points fixes et de transpositions, et de ces deux transformations seulement.

Le volet suivant consiste à pondérer les fixes par x et les transpositions par (-1), puis à pondérer toute involution $\sigma$ par son poids $\mu(\sigma)$ égal à $(-1)^{\operatorname{trans}(\sigma)} x^{\text {fix }(\sigma)}-$ où fix $(\sigma)$ (resp. $\operatorname{trans}(\sigma))$ est le nombre de points fixes (resp. de transpositions) dans $\sigma$, et où $x$ est un paramètre réel muet «générateur ». Dans ces conditions, par un décompte effectué à partir de (2), D. Foata établit que la valeur du polynôme d'Hermite $H_{n}(x)$ coincide avec la somme de tous les $\mu(\sigma)$ où $\sigma$ décrit l'ensemble Inv $n$ de toutes les involutions d'ordre $n$, c'est-à-dire $H_{n}(x)=\sum_{\sigma \in \operatorname{Inv}_{\mathrm{n}}} \mu(\sigma)$ ce qu'il note naturellement $\mu\{\operatorname{Inv} n\}$. Ainsi le membre de gauche est-il réinterprété selon $\sum_{n \geq 0} \frac{u^{n}}{n !} \mu\left\{\operatorname{Inv}_{n}\right\}$.

Quant au membre de droite, c'est tout simplement l'exponentielle d'un polynôme du second degré $\left(u x-\frac{u^{2}}{2}\right)$. Cette bien simple expression va être pourtant complexifiée 
pour être considérée comme la somme d'une série entière $\sum_{n \geq 1} t_{n} \frac{u^{n}}{n !}$ avec évidemment $t_{1}=x, t_{2}=-1$ et $t_{k}=0$ pour $k \geq 3$.

Identifiant en effet, comme on l'a dit, toute involution de $[n]$ avec son graphe à $\mathrm{n}$ sommets, Foata fait immédiatement constater que « les seules composantes connexes du graphe [d'une involution] sont les boucles, de poids $x$ et, ou les cycles de longueur 2, de poids $-1 \gg$ selon

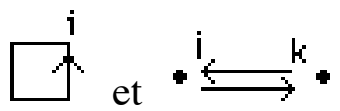

En renumérotant «canoniquement» (i.e à partir de 1) les fixes et les transpositions, on a donc deux schémas possibles seulement :

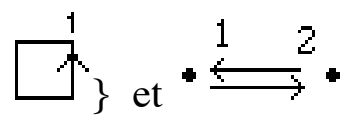

On comprend alors bien comment, d'une part les involutions connexes engendrent toutes les involutions, comment d'autre part aussi la fonction de poids $\mu$ s'applique naturellement aux involutions connexes. Il suffit alors d'envisager, pour chaque $n$, l'ensemble $\operatorname{Invc}_{n}$ de toutes les involutions connexes d'ordre $n$ (c'est-à-dire dont le graphe est connexe), puis le poids $\mu\left\{\operatorname{Invc}_{n}\right\}$ de cet ensemble. On a

$$
\operatorname{Invc}_{1}=\{\stackrel{1}{\stackrel{1}{S}}\} ; \operatorname{Invc}_{2}=\left\{\stackrel{1 \stackrel{2}{\leftrightarrows}^{2}}{\leftrightarrows}\right\} ; \operatorname{Invc}_{k}=\varnothing \text { pour } k \geq 3
$$

Ainsi $\mu\left\{\operatorname{Invc}_{1}\right\}=x, \mu\left\{\operatorname{Invc}_{2}\right\}=-1$ et $\mu\left\{\operatorname{Invc}_{k}\right\}=0$ pour $k \geq 3$. Il est clair que l'on ainsi retrouve les $t_{k}$ précités. Et donc

$$
\sum_{n \geq 1} \frac{u^{n}}{n !} \mu\left\{\operatorname{Invc}_{n}\right\}=u \mu(\stackrel{1}{\square})+\frac{u^{2}}{2} \mu\left(\stackrel{1 \stackrel{2}{\leftrightarrows}^{2}}{\leftrightarrows}\right)+0=u x-\frac{u^{2}}{2}
$$

On constate en même temps qu'il aurait encore suffi de définir $\mu$ sur les involutionnaires connexes pour en disposer sur tous les involutionnaires. Dans ces conditions cependant, le terme de droite $\exp \left(u x-\frac{u^{2}}{2}\right)$ de la formule-pivot s'interprète aussi comme le résultat d'une énumération et le pivot se réécrit suivant

$$
\sum_{n \geq 0} \frac{u^{n}}{n !} \mu\left\{\operatorname{Inv}_{n}\right\}=\exp \left(\sum_{n \geq 1} \frac{u^{n}}{n !} \mu\left\{\operatorname{Invc}_{n}\right\}\right)
$$

Comme l'écrit excellement l'auteur,

la fonction génératrice exponentielle des polynômes générateurs des involutions sur les intervalles [n] est égale à l'exponentielle de la fonction génératrice exponentielle des polynômes générateurs des involutions connexes sur ces mêmes intervalles.

Le deuxième temps logique de l'article consiste à ne pas se satisfaire du (pourtant remarquable) résultat précédent, mais à l'interpréter à son tour comme une instance 
seulement d'une formule paradigmatique bien plus large, dénommée par D. Foata identité exponentielle

$$
\sum_{n \geq 0} \frac{u^{n}}{n !} \mu\left\{Y_{n}^{+}\right\}=\exp \left(\sum_{n \geq 1} \frac{u^{n}}{n !} \mu\left\{Y_{n}\right\}\right)
$$

où $Y=\left(Y_{n}\right)(n \geq 1)$ est une suite d'ensembles finis quelconques (dans l'exemple, les graphes involutionnaires ) et $Y^{+}=\left(Y_{n}^{+}\right)$une nouvelle suite d'ensembles construits à partir de $Y$, appelée composé partitionnel de $Y$ (resp. les graphes involutionnaires connexes).

La question (et la difficulté) était en vérité d' abstraire de l'exemple involutionnel ci-dessus une conceptualisation générale adéquate - ce qui n'était pas si simple. La méthode de Foata consiste en sa remarquable construction algebrico-combinatoire des composés partitionnels, à la fois générale et centrale, qui se définit, comme lui-même l'écrit par ailleurs ${ }^{4}$, de permettre de ramener «une construction d'énumération à un problème analogue sur une sous-famille «génératrice» constituée par des objets «connexes ». C'est une méthode véritablement très générale dont il est sans doute ici plus simple, pour poursuivre notre commentaire méthodologique, d'utiliser pour l'exposer un autre article de D. Foata A combinatorial proof for Mehlers's formula et prendre ainsi pour appui «pédagogique » la décomposition d'un graphe fini quelconque (et non plus nécessairement involutionnaire) $G$ (non orienté, dont $X$ est l'ensemble des $n$ sommets) en ses $r$ composantes connexes $\left\{G_{1}, \ldots, G_{r}\right\}$. Chaque composante connexe $G_{i}$ (dont $I_{i}$ est l'ensemble des sommets) est elle-même retranscrite, après renumérotation de ses sommets pour retrouver chaque fois l'ordre naturel comme un certain couple $\left(y_{i}\right.$, $I_{i}$ ) où $y_{i}$ est un graphe connexe (en un certain sens «isomorphe» à $G_{i}$ ) dont les sommets sont étiquetés «naturellement» $\left(1,2, \ldots, \operatorname{Card}\left(G_{i}\right)\right)$. Un tel couple est une « indéterminée ». De son côté, la suite :

$$
w=\left(y_{1}, I_{1}\right)\left(y_{2}, I_{2}\right) \ldots\left(y_{r}, I_{r}\right)
$$

obtenue par juxtaposition (commutative) d'indéterminées est un « monôme » - en vérité un élément d'un certain monoïde abélien libre. Il y a cependant des contraintes : d'une part on doit évidemment avoir $\operatorname{Card}\left(y_{i}\right)=\operatorname{Card}\left(I_{i}\right)$; d'un autre côté, l'ensemble, des secondes composantes $I_{k}$ est toujours astreint à constituer chaque fois une partition de $X$. Dans ces conditions cependant, on a dans chaque première composante $y_{k}$, « remis les pendules à l'heure » à partir de $G_{k}$, c'est-à-dire l'origine à 1 et respecté la numérotation qui s'ensuit ${ }^{5}$. La donnée de $I_{k}$ permet ensuite le « raccord» avec le graphe initial. On reconnaît les mécanismes de l'exemple involutionnaire supra. Ainsi l'écriture chez Foata d'une indéterminée $\left(y_{i}, I_{i}\right)$ distingue-t-elle ultimement la structure, représentée par $y_{i}$ (qui dit de quel type de sous-graphe il s'agit) de la localisation, représentée par $I_{i}$ (c'est-à-dire de la place de ce sous-graphe dans le graphe total $G$ ). Une séparation qui est décisive pour une représentation adéquate.

La suite finie w est alors équivalente à la donnée du graphe $G$. À l'ensemble $Y_{n}$ de tous les graphes à $n$ sommets est alors associé l'ensemble $Y_{n}^{+}$de tous les monômes $w$ satisfaisant aux contraintes précédentes(c'est le composé partitionnel à l'ordre $n$ ). Lorsque $n$ varie, à l'ensemble $Y=\left\{Y_{n} ; n \geq 1\right\}$ de tous les graphes finis, est ainsi associé

\footnotetext{
${ }^{4}$ D. Foata et M.-P. Schützenberger, « Théorie géométrique des polynômes eulériens », Lectures Notes in Math., ${ }^{\circ} 138$, Springer-Verlag, Berlin, 1971, ici p. 6.

${ }^{5}$ Les arêtes et les boucles de $y_{k}$ « suivant la retranscription » à partir de celles de $G_{k}$.
} 
l'ensemble $Y^{+}=\left\{Y_{n}^{+} ; n \geq 1\right\}$ qui est le composé partitionnel (abélien) de $Y$. Il est bien clair sur cet exemple des graphes que la définition de $Y_{n}^{+}$fait intervenir tous les $Y_{k}$ avec $k \leq n$ et que la correspondance doit ainsi être, non pas terme à terme, mais bien suite à suite, $Y \rightarrow Y^{+}-$le procédé était bien celui déjà appliqué supra aux involutionnaires.

Dépassant encore le cas particulier des graphes, cette définition, mise à jour par D. Foata, subtile et profonde en vérité, sera ensuite appliquée par son auteur à toute suite $Y=Y_{n}$ d'ensembles finis pour en concevoir de façon générale le $Y^{+}$associé. On définit ainsi successivement les indéterminées, les monômes, le composé partitionnel à l'ordre $n$, enfin le composé partitionnel $Y^{+}$(réunion de tous les précédents). D'autres exemples conduiront D. Foata à s'interroger sur le composé partitionnel $F^{+}$de l'ensemble $F$ de toutes les applications entre ensembles finis, ou bien sur $P^{+}$, composé partitionnel de l'ensemble $P$ de toutes les permutations (substitutions d'ensembles finis) $\left(P=\left\{P_{n} ; n \geq 1\right\}\right)$. Chaque fois, les éléments de $F^{+}$(resp. de $\left.P^{+}\right)$apparaîtront ainsi, en un certain sens, comme des «générateurs connexes» de ceux de $F$ (resp. de $P$ ) ; ainsi par exemple des permutations circulaires relativement aux permutations quelconques.

Le composé partitionnel $Y^{+}$de $Y$ ainsi organisé, le second volet de la méthode, chaque fois spécifique d'un dénombrement particulier, consiste à définir comme plus haut une fonction $\mu$ sur $Y^{+}$(en vérité donc sur les seules parties «atomiques » de $Y$, i.e ici connexes). à valeurs dans une algèbre quelconque de polynômes, par exemple IR[X]. Définie sur les monômes, la fonction de poids $\mu$ l'est naturellement sur les indéterminées $(y, I)$. Deux contraintes adéquates - simplement transposées des conditions vérifiées dans l'exemple involutionnaire - sont alors imposées sur elle pour la rendre «multiplicative »- on constate alors simplement qu'il suffisait de définir $\mu$ sur les indéterminées.

Le théorème combinatoire fondamental de D. Foata est alors celui-ci, en forme de paradigme : en injectant les deux suites supra $\mu\left(Y_{n}^{+}\right)$et $\mu\left(Y_{n}\right)$ comme coefficients de deux développements en séries exponentiels

$$
a=1+\sum_{n \geq 1} \frac{u^{n}}{n !} \mu\left\{Y_{n}^{+}\right\} \quad \text { et } \quad b=\sum_{n \geq 1} \frac{u^{n}}{n !} \mu\left\{Y_{n}\right\}
$$

les fonctions génératrices obtenues qui sont - en sens inverse - les sommes de ces deux développements sont liées par une relation du type $a=\exp (b)$ selon

$$
1+\sum_{n \geq 1} \frac{u^{n}}{n !} \mu\left\{Y_{n}^{+}\right\}=\exp \left(\sum_{n \geq 1} \frac{u^{n}}{n !} \mu\left\{Y_{n}\right\}\right)
$$

Toute analyse d'un phénomène combinatoire en termes de $Y$ et de $Y^{+}$conduit ainsi à une relation du type $a=\exp (b)$ (ou encore $b=\log (a)$ ) entre fonctions génératrices exponentielles associées. Réciproquement, toute formule d'analyse entre deux développements en série qui peut se mettre sous la forme $a=\exp (b)$ peut être recherchée comme témoignant de la constitution du composé partitionnel $Y^{+}$d'une suite $Y$ d'ensembles finis. Il s'agit évidemment dans chaque cas de rechercher et construire un $Y$ adéquat, d'en reconnaître le $Y^{+}$et d'adapter une fonction de poids $\mu$ au problème considéré. Ainsi en cas de succès a-t-on obtenu une démonstration du résultat d'analyse considéré qui est, non pas seulement combinatoire, mais bien «paradigmatiquement » 
combinatoire. Les deux formules précitées concernant les polynômes d'Hermite relèvent directement de cette figure de pensée.

Le troisième temps de l'article consiste en effet à réinterpréter à son tour combinatoirement la formule de Mehler (3) pour la faire entrer dans le cadre «partitionnel», en utilisant à nouveau des graphes particuliers, ici dits biinvolutionnaires. Le membre de gauche («bilinéaire ») $\sum_{n \geq 0} \frac{u^{n}}{n !} H_{n}(x) H_{n}(y)$ s'interprète certes immédiatement comme précédemment selon $\sum_{n \geq 0} \frac{u^{n}}{n !} \mu\left\{\operatorname{In} v_{n} \times \operatorname{In} v_{n}\right\}$, en considérant les couples d'involutions - $\mu$ étant défini de façon structurellement analogue. Il reste à réinterpréter le membre de droite $\left(1-u^{2}\right)^{-1 / 2} \exp \left(\frac{2 u x y-u^{2}\left(x^{2}+y^{2}\right.}{2\left(1-y^{2}\right)}\right)$ qui est, après développement de $\left(1-u^{2}\right)^{-1 / 2}$, encore une exponentielle (formule (5.2) de l'article). Comme dans le cas du pivot, l'argument de cette dernière est retransformé en considérant cette fois l'ensemble produit $\operatorname{Inv}_{n} \times \operatorname{Inv}_{n}$, c'est-à-dire les couples d'involutions, ou - ce qui revient au même - les graphes de ces couples, dits biinvolutionnaires, puis en caractérisant parmi eux les sous-graphes (bi-involutionnaires) connexes dont l'article fait l'inventaire, montrant qu'ils sont constitués, pour chaque $n$, comme une certaine réunion disjointe $Y_{n}$ de quatre types de termes. Le résultat crucial, ici remarquablement expliqué sur un exemple de graphes, est que l'ensemble des graphes bi-involutionnaires connexes est le composé partitionnel $Y^{+}=\left(Y_{n}^{+}\right)$de la suite $Y=\left(Y_{n}\right)$ des graphes bi-involutionnaires. La formule de Mehler est donc bien elle aussi une instance du paradigme. Le texte montre enfin qu'il en est de même pour la formule multilinéaire. 